\title{
Bayesian Design of Plasma Diagnostics
}

\author{
H. Dreier, A. Dinklage, R. Fischer*, M. Hirsch, P. Kornejew \\ Max-Planck-Institut für Plasmaphysik, EURATOM Association, \\ Teilinstitut Greifswald, D-174891 Greifswald, Germany \\ * Boltzmannstr. 2, D-85748 Garching, Germany
}

\begin{abstract}
A framework for diagnostics design based on Bayesian probability theory is introduced. The design is assessed in figures of an expected utility which measures the information gain from measurements. The approach is applied on design studies for a multi-chord interferometer for Wendelstein 7-X.
\end{abstract}

\section{DESIGN OF PLASMA DIAGNOSTICS}

The design of plasma diagnostics is a typical task to be resolved along the preparation of fusion experiments. Requirements for a design process are

- highest accuracy of measurements

- high resolution $(t, \vec{x})$

- robustness

- extensibility

In addition, the design process has to meet with constraints such as accessibility or economic restrictions.

A typical design process consists of a number of considerations based on typical use cases or - synonymously - set of assumptions on the expected physical scenarios and possibilities for the technical realization of the measurement. In a real experiment, however, the data produced by the diagnostics are ultimately employed for the resolution of physical issues, or in other words the quantitative assessment of hypotheses.

One can conclude that - as the analysis of data - the design of plasma diagnostics is a complicated task which includes all aspects of plasma physics and the analysis of noisy and often incomplete data. But the design task exhibits two more challenges, that is

1. one has to deal with expected data of a future experiment and
2. one needs to quantify the benefit of a design consideration.

The latter point allows one for design decisions (e.g. is it justified to add an additional laser beam in a multichord interferometer).

Consequently, the design process is generally considered to be an intricate problem and many (correct) design decisions are based on experience from former set-ups. Since the overall process requires the inclusion of many different aspects, approaches to quantify the expected utility of a diagnostics are beneficial for design considerations.

This paper summarizes a framework which addresses the issues raised and provides a mathematical formulation to support the design process. Results for an application on design considerations of the interferometer set-up for Wendelstein 7-X are shown in [1].

\section{DESIGN STRATEGY}

Fig. 1 shows a simplified work-flow of the overall design procedure. The essential part is the optimization of the expected utility of the set-up with respect to design parameters (e.g. the chord position)

In order to formulate the design procedure as an optimization problem, the quantities to be measured are to be specified. Beyond crude estimates of ranges, it is intended to employ predictive modelling for W7-X specific physical issues, e.g. confinement studies. This philosophy is reflected by the choice 


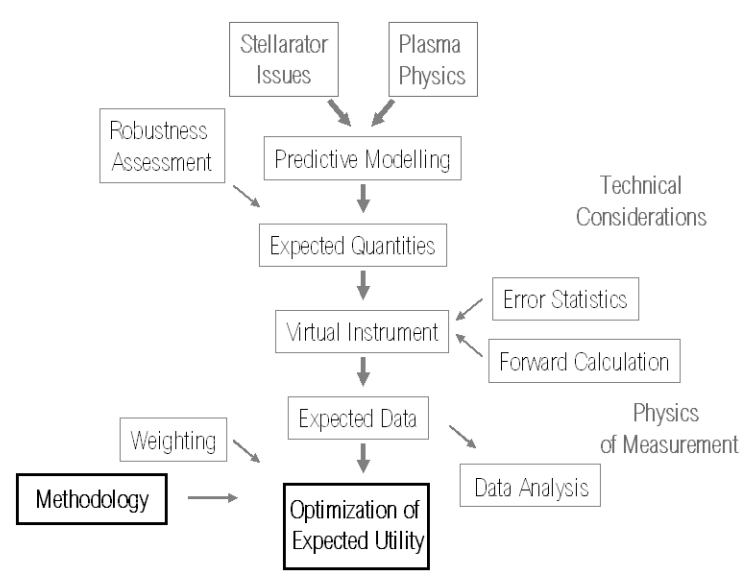

FIG. 1: Elements of the design procedure.

of "start-up" diagnostics for Wendelstein 7-X which is oriented to resolve physical issues of stellarator optimization [2]. In assistance, the robustness of the results is assessed by any available additional information, e.g. global scaling laws in the case of confinement studies. The resulting expected quantities are fed to a virtual instrument which implements the forward model of the measurement and an essential part - describes the error statistics (likelihood). For the range of expected data, the optimization procedure may also include a weighting with respect to the quantity ranges of interest. Technical and physical considerations enter the process at different stages. For a proof of results the analysis of data from the virtual instrument is part of the design strategy.

\section{BAYESIAN EXPERIMENTAL DESIGN}

Lindley [3] proposed an approach based on decision theory to design. It begins with the choice of an appropriate utility function reflecting purpose and costs of an experiment. For the quantification of the utility of a setup, the Kullback-Leibler distance $U_{K L}$ measures the information gain from the ignorance on a quantity $\phi$ before a measurement to the knowledge after data $D$ are taken:

$$
\begin{array}{r}
U_{K L}(D, \eta)=\int P(\phi \mid D, \eta) \\
\cdot \log \left(\frac{P(\phi \mid D, \eta)}{P(\phi)}\right) \mathrm{d} \phi
\end{array}
$$

It is measured in bits, if the base-2 logarithm is used.

Uncertainties are encoded as probability density functions $P$. The conditional probability $P(\phi \mid D)$ means the probability that $\phi$ is true given the data $D$. Please note, that $U_{K L}$ depends both on the data $D$ and the design parameters $\eta$ which are the optimization parameters.

An integration over the range of expected data, where the evidence of the data is represented by the probability density function $P(D \mid \eta)$, yields the expected utility function $E U$ :

$$
E U(\eta)=\int P(D \mid \eta) U_{K L}(D, \eta) \mathrm{d} D
$$

which is a function of the design parameters $\eta$ only. It is a measure of the mean information gain from the data, averaged over the expected data space.

The principle of Bayesian diagnostic design ist the maximization of the $E U$ with respect to $\eta$.

Using Bayes theorem

$$
P(\phi \mid D, \eta)=\frac{P(D \mid \phi, \eta) \cdot P(\phi)}{P(D \mid \eta)}
$$

the $E U$ is given by

$$
\begin{gathered}
E U=\iint P(\phi) P(D \mid \phi, \eta) \\
\cdot \log \left(\frac{P(D \mid \phi, \eta)}{P(\phi)}\right) \mathrm{d} \phi \mathrm{d} D .
\end{gathered}
$$

This formulation uses the likelihood $P(D \mid \phi, \eta)$ and a probability density function $P(\phi)$ which reflects the range of interest and weighting for $\phi$.

The likelihood can be regarded as a representation of a diagnostics model. It contains 
the forward calculation and the error statistics of the forward model parameters. The expected utility is now a function of the design parameters $\eta$ only and is subject of optimization studies [4].

\section{OPTIMIZATION STUDIES FOR THE W7-X INTERFEROMETER}

The error statistics of the measurement has crucial impact on the expected utility [5]. For the examples presented here, a constant error is chosen which is about a few percent, depending on the actual data value.

For the creation of virtual data, a parametrized density function is used:

$$
n(r)=\phi_{1} \cdot \frac{1+\phi_{4} \cdot\left(r / r_{\max }\right)^{2}}{1+\left(\frac{\left(r / r_{\max }\right)^{2}}{\phi_{2}^{2}}\right)^{\phi_{3}}}
$$

The parameters $\phi_{1} \ldots \phi_{4}$ represent the maximum density, position of the edge gradient, steepness and bulge of the density distribution.

Fig. 2 shows the expected utility for a single beam, where the optimization target is the reconstruction of each parameter $\phi_{i}$. The result is displayed in figures of two angles $\eta_{1}$ and $\eta_{2}$ which represent the starting and end point of a chord on a circumventing circle as indicated in Fig. 3.

The results indicate the different impact of shaping. Coarsely, the chord represents the maximum signal-to-noise ratio (SNR) chord for the respective parameter. For the maximum (Fig. 2(a)) a beam traversing the plasma center yields best SNR. Since the shaping effects of the other parameters are most distinct at the plasma edge, the resultant reconstruction has maximum information gain for sightlines traversing the edge region. The parameters steepness and bulge have nearly coinciding optimum chords as to be seen in Fig. 3 where the outmost beam is degenerated (bulge, steepness).

The benefit of this approach is a quantification of the design quality which also allows

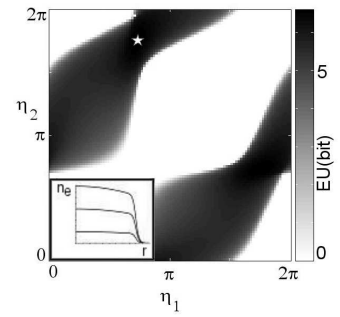

(a)

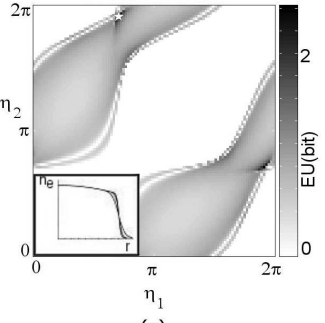

(c)

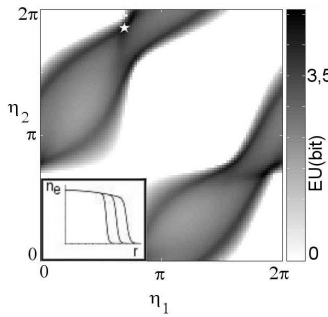

(b)

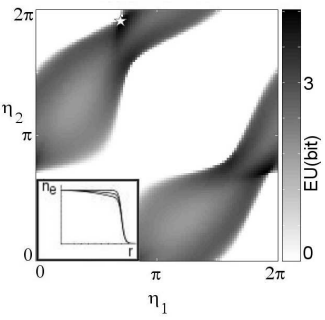

(d)
FIG. 2: Expected Utility for estimation of maximum density (a), gradient position (b), steepness (c) and bulge (d). The data space is generated by a variation of (a) $\phi_{1}=0 \ldots 5 \times 10^{20} \mathrm{~m}^{-3}$, (b) $\phi_{2}=0.6 \ldots 0.95$, (c) $\phi_{3}=1 \ldots 30$ and (d) $\phi_{4}=-1 \ldots 0$. The star symbol marks the maximum of the EU. The insets show the corresponding density profile variation where the maximum ordinate is $n_{e}=1 \times 10^{20} \mathrm{~m}^{-3}$ and the abscissae are effective radii $\left(r_{e f f} / a\right)$.

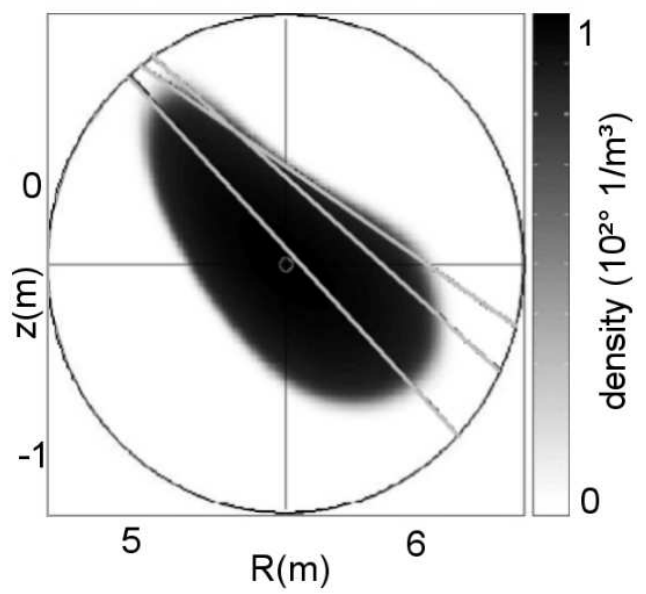

FIG. 3: Density distribution in the toroidal plane for the W7-X interferometer. The chords shown represent the optimum reconstruction of data (cf. 2). The center beam corresponds to Fig. 2(a), the outmost beam to Figs. 2(c) and (d). 
the diagnostician to estimate the robustness of a design chosen.
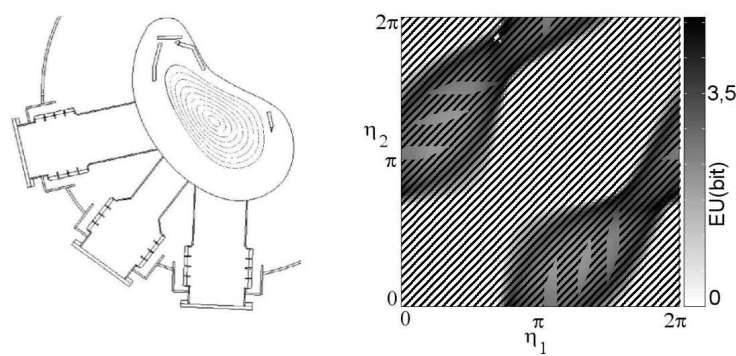

FIG. 4: Restricted access to the plasma due to port system (left), accessible chord positions in EU plot for the gradient position case (lightened areas, right).

Technical constraints arise due to restricted access to the plasma vessel through the ports. Fig. 4 indicates accessible chords in figures of the parametrization chosen.

The effect of the technical restrictions can be quantified and compared to ideal access.
A translation of the expected utility to measurement uncertainties is straightforward but depends on the forward function of the virtual instrument.

\section{DISCUSSION}

Bayesian diagnostics design is applied on plasma diagnostics. The design allows for a quantification of design considerations and estimates for their robustness. The reconstruction of density profiles by means of a multichannel infrared interferometer at Wendelstein 7 -X shows how measuring capabilities can be detected and complicated entanglements of measurement and geometry revealed. The impact of technical boundary conditions can be quantified as well as the information gain by the inclusion of additional chords.
[1] P. Kornejew, M. Hirsch, T. Bindemann, A. Dinklage, H. Dreier, H.-J. Hartfuß, Rev. Sci. Instrum. (these proceedings).

[2] H.J. Hartfuß et al., Rev. Sci. Instrum. 68, 1244 (1997)

[3] Lindley, D. V. Bayesian Statistics - A Review (SIAM, Philadelphia, 1972).

[4] R. Fischer, in Bayesian Inference and Maxi- mum Entropy Methods in Science and Engineering, AIP Conference Proc. 735, 76 (2004).

H. Dreier, A. Dinklage, R. Fischer, M. Hirsch, P. Kornejew, E. Pasch, Fusion Sci. Technol. 50, 262 (2006). 